\title{
Low Molecular Weight Heparin Nebulization Attenuates Acute Lung Injury
}

\author{
Nianlin Xie, ${ }^{1}$ Menglei Huan, ${ }^{2}$ Feng Tian, ${ }^{1}$ Zhongping Gu, ${ }^{1}$ and Xiaofei Li ${ }^{1}$ \\ ${ }^{1}$ Department of Thoracic Surgery, Tangdu Hospital, The Fourth Military Medical University, Xian 710038, China \\ ${ }^{2}$ Department of Pharmaceutics, School of Pharmacy, The Fourth Military Medical University, Xian 710032, China \\ Correspondence should be addressed to Zhongping Gu; guzhongpingtd@163.com and Xiaofei Li; lixiaofeitd@163.com
}

Received 3 November 2016; Revised 11 March 2017; Accepted 14 March 2017; Published 15 May 2017

Academic Editor: Vasiliki Galani

Copyright (c) 2017 Nianlin Xie et al. This is an open access article distributed under the Creative Commons Attribution License, which permits unrestricted use, distribution, and reproduction in any medium, provided the original work is properly cited.

\begin{abstract}
Background. As acute lung injury (ALI) caused high mortality rate, it is important to explore the protection and treatment of ALI. The aim of the current study is to evaluate the effect of low molecular weight heparin (LMWH) nebulization on attenuating acute lung injury and the associated mechanism. Methods. The arterial blood gas, total protein content in bronchoalveolar lavage fluid, lung wet/dry weight ratio, malondialdehyde (MDA) content, superoxide dismutase (SOD) and glutathione peroxidase (GSHPx) activity, and Akt phosphorylation were evaluated after the ALI rabbits were treated with or without LMWH nebulization. Results. $\mathrm{PaO}_{2}$ was increased and lung wet/dry weight ratio as well as total protein content in BALF was decreased after LMWH nebulization. After the application of LMWH nebulization therapy, the SOD and GSH-Px activity was rebounded and the increase of MDA content was significantly inhibited. The Akt protein phosphorylation level was decreased after LMWH nebulization therapy. Conclusions. LMWH nebulization treatment can relieve the traumatic ALI in rabbits and inhibit oxidative stress possibly by suppressing the Akt phosphorylation.
\end{abstract}

\section{Introduction}

Acute lung injury (ALI) is caused by a variety of lung damaging disease. Refractory hypoxemia and respiratory distress are the main clinical features of ALI. Many studies have reported that ALI has a mortality rate of 40 to 60 percent [1]. Due to its high mortality rate, exploring the protection and treatment of ALI is getting more and more attention of the people. It is reported that ALI is a set of clinical syndromes with pathological basis of increased vascular permeability, fibrin deposition, and a large number of edema fluid accumulations inside alveoli [2]. ALI is characterized of inflammatory mediator releasing, blood coagulation, and fibrinolysis dysfunction $[3,4]$.

As is known to all, heparin is an important anticoagulant and has already been widely used as anticoagulant agent clinically. The anticoagulant effect of heparin in vivo or in vitro is very strong. In addition, heparin can inhibit inflammation, improve microcirculation, regulate blood coagulation disorders, and may therefore possibly have a certain therapeutic effect against ALI. By contrast, low molecular weight heparin (LMWH) is a kind of heparin produced by nitrous acid decomposition and purification of low molecular heparin sodium or calcium salts. LMWH has the function of fast and continuous thrombosis resistance and can improve hemodynamics. It has higher bioavailability, longer half-life, and lower bleeding risk than ordinary heparin so it is more secure and easier to use [5].

In recent years, more and more research focused on heparin therapy on ALI. Han et al. reported that unfractionated heparin may attenuate endotoxin-induced lung vascular leak in ALI model of mice [6]. Mu et al. noted that heparin significantly ameliorated the lung injury induced by LPS in rats and may be a potential therapeutic reagent for treating ALI in the future [7]. Wang et al. found that the antiinflammatory mechanisms of heparin in ALI may alleviate the inflammatory reaction in rabbits [8].

Although the previous studies discussed and put forward the effect of heparin in the treatment of ALI, they adopted the heparin intravenously. There is a possibility that intravenous administration may reduce the local function of heparin and cause the whole body adverse effects such as systemic 
complications. In order to avoid these problems, we propose to study the effects of LMWH by local nebulization delivery in the treatment of ALI in the current study.

In order to explore the therapeutic effects and the related mechanism of LMWH nebulization on ALI, we observed the arterial blood gas, total protein content in bronchoalveolar lavage fluid, lung wet/dry weight ratio, malondialdehyde (MDA) content, superoxide dismutase (SOD) and glutathione peroxidase (GSH-Px) activity, and Akt phosphorylation after establishing the model of ALI rabbits.

\section{Materials and Methods}

2.1. ALI Model. Before the induction of ALI, rabbits were fasted overnight but allowed water ad libitum. Animals in experimental groups were anesthetized with intraperitoneal pentobarbital $(50 \mathrm{mg} / \mathrm{kg})$ and were impacted on the right chest with driving-velocity at the following parameters: velocity $20 \mathrm{~m} / \mathrm{s}$, compression $20 \%$, and weight $5.7 \mathrm{~kg}$ to develop ALI model. All animal protocols were approved by the Committee on Animal Investigations of Fourth Military Medical University.

2.2. Treatment Groups. Twenty-four healthy male New Zealand white rabbits (supplied by the Experimental Animal Center of Fourth Military Medical University, Xian, Shaanxi, China), weighing 2.5 to $3.2 \mathrm{~kg}$, were divided into three groups at random $(n=8$ each): control group in which animals were exposed to room air in the same cabinet; saline nebulization group in which animals were repetitively administered with saline solution (immediately after being impacted on the right chest and subsequently every $4 \mathrm{~h}$ thereafter) by ultrasonic nebulization via a tightly fitting mask and under spontaneous breathing; and low molecular weight heparin nebulization group in which animals were repetitively administered with low molecular weight heparin $(1 \mathrm{U} / \mathrm{g}$, immediately after being impacted on the right chest and subsequently every $4 \mathrm{~h}$ thereafter) by ultrasonic nebulization via a tightly fitting mask and under spontaneous breathing.

2.3. Arterial Blood Gas Analysis. Arterial blood was collected at 24 hours after ultrasonic nebulization for arterial blood gas analysis.

2.4. Total Protein Content in Bronchoalveolar Lavage Fluid. Animals were anesthetized with intraperitoneal pentobarbital $(50 \mathrm{mg} / \mathrm{kg})$. A median sternotomy allowed for exposure of both of the lungs. The trachea was exposed and inserted with an intravenous infusion needle. After ligating the hilum of the right lung, the left lung was lavaged 5 times with $0.5 \mathrm{~mL}$ icecold phosphate buffered saline. The recovery ratio of the fluid was about $90 \%$. The bronchoalveolar lavage fluid (BALF) was immediately centrifuged at $500 \mathrm{~g}$ for $10 \mathrm{~min}$ at $4^{\circ} \mathrm{C}$, and the cell-free supernatant was stored at $-80^{\circ} \mathrm{C}$ for analysis of the content of total protein in BALF.

2.5. Lung Wet/Dry Weight Ratio. As an index of lung edema, the amount of extravascular lung water was calculated. The middle lobe of the right lung was excised and the wet weight was recorded. The lobe was then placed in an incubator at $80^{\circ} \mathrm{C}$ for $24 \mathrm{~h}$ to obtain the dry weight. And the wet/dry weight ratios were calculated by dividing the wet weight by the dry weight.

2.6. Malondialdehyde (MDA) Content as Well as Superoxide Dismutase (SOD) and Glutathione Peroxidase (GSH-Px) Activity Assay. The lungs were homogenized in ice-cold phosphate buffered saline and centrifuged at $500 \mathrm{~g}$ for $10 \mathrm{~min}$ at $4^{\circ} \mathrm{C}$. The supernatant was stored at $-80^{\circ} \mathrm{C}$ for analysis of MDA content and activity of SOD and GSH-Px with test kits (Nanjing Jiancheng Bioengineering Institute, Nanjing, Jiangsu, China) according to kit instructions.

2.7. Immunoblot Analysis. The lungs were homogenized in lysis buffer (20 mM HEPES pH 7.4, 1\% Triton X-100, 10\% glycerol, $2 \mathrm{mM}$ ethylene glycol-bis (b-aminoethyl ether)$\mathrm{N}, \mathrm{N}, \mathrm{N}^{\prime}, \mathrm{N}^{\prime}$-tetraacetic acid, $50 \mu \mathrm{M}$ b-glycerophosphate, $1 \mathrm{mM}$ sodium orthovanadate, $1 \mathrm{mM}$ dithiothreitol, $400 \mu \mathrm{M}$ aprotinin, and $400 \mu \mathrm{M}$ phenylmethylsulfonyl fluoride), transferred to Eppendorff tubes, and placed on ice for 15 minutes. Tubes were centrifuged at $14,000 \mathrm{rpm}$ for 10 minutes at $4^{\circ} \mathrm{C}$ and supernatant was flash frozen. Crude cell lysates were matched for protein concentration, resolved on a $10 \%$ bisacrylamide gel, and electrotransferred to Immobilon-P membranes (Millipore Corp., Bedford, MA, USA). For assay of Akt phosphorylation and Akt total protein expression, Western blot analyses were performed with antibodies of phospho-Akt and Akt (Cell Signaling Biotechnology, Beverly, MA, USA). Blots were developed by enhanced chemiluminescence (NEN Life Science Products, Boston, MA, USA).

2.8. Statistical Analysis. Data of each group were expressed as mean \pm standard deviation (SD). Results were processed by variance analysis and $t$-test with the software of SPSS 13.0. Statistical significance was set at $P<0.05$.

\section{Results}

3.1. Effects of LMWH Nebulization on Partial Pressure Arterial Oxygen $\left(\mathrm{PaO}_{2}\right)$. In saline nebulization group, the partial pressure arterial oxygen $\left(\mathrm{PaO}_{2}\right)$ was significantly decreased compared with control group $(P<0.05)$. However, low molecular weight heparin nebulization attenuated this decrease in $\mathrm{PaO}_{2}$ (Figure 1). Although $\mathrm{PaO}_{2}$ in LMWH nebulization group was still lower than that in control group $(P<0.05)$, $\mathrm{PaO}_{2}$ was significantly higher after LMWH nebulization than that in saline nebulization group $(P<0.05)$.

\subsection{Effects of LMWH Nebulization on Total Protein Content} in BALF. Compared with control group, the content of total protein in BALF in both saline nebulization group and low molecular weight heparin nebulization group increased significantly $(P<0.05$, Figure 2$)$, while content of total protein in BALF in LMWH nebulization group decreased significantly compared to saline nebulization group $(P<0.05$, Figure 2). 


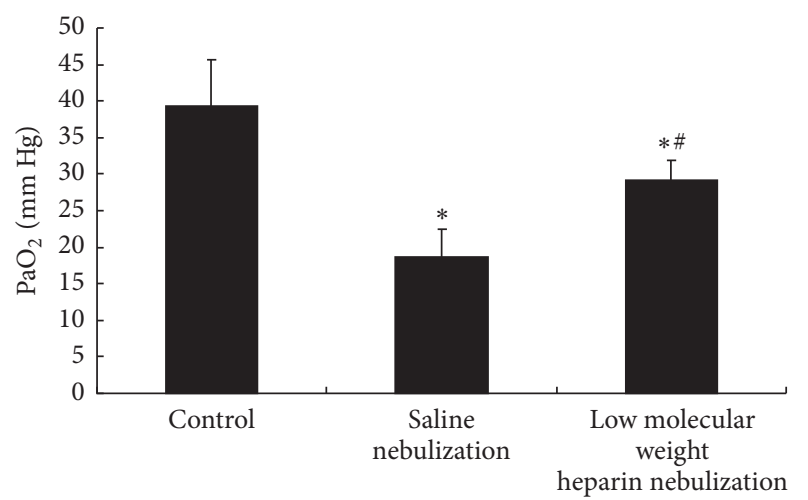

FIGURE 1: Effects of low molecular weight heparin nebulization on partial pressure arterial oxygen $\left(\mathrm{PaO}_{2}\right)$. Arterial blood was collected at 24 hours after ultrasonic nebulization for arterial blood gas analysis. Data are presented as the mean \pm SD. ${ }^{*} P<0.05$ versus control group; ${ }^{\#} P<0.05$ versus saline nebulization group.

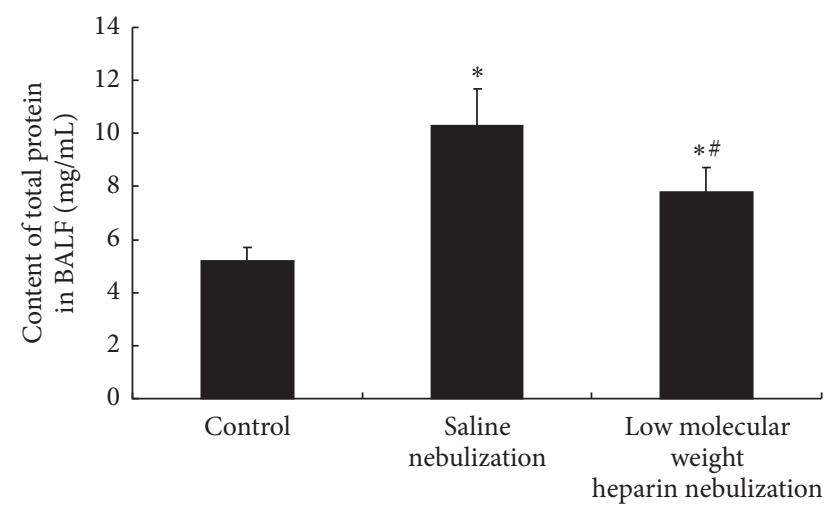

FIGURE 2: Effects of low molecular weight heparin nebulization on content of total protein in bronchoalveolar lavage fluid (BALF). After the animals were anesthetized, the left lung was lavaged 5 times with ice-cold phosphate buffered saline. The BALF was centrifuged and the supernatant was analyzed for the content of total protein in BALF. Data are presented as the mean $\pm \mathrm{SD} .{ }^{*} P<0.05$ versus control group; ${ }^{\#} P<0.05$ versus saline nebulization group.

3.3. Effect of LMWH Nebulization on Lung Wet/Dry Weight Ratio. As shown in Figure 3, the lung wet/dry weight ratio in saline nebulization group was significantly increased compared with control group $(P<0.05)$. The increase in lung wet/dry weight ratio was significantly reduced by LMWH nebulization $(P<0.05)$.

3.4. Effects of LMWH Nebulization on MDA Content, SOD, and GSH-Px Activity. Compared with control group, activity of SOD and GSH-Px in saline nebulization group was significantly decreased $(P<0.05$, Figures 4 and 5), and content of MDA in saline nebulization group was significantly increased $(P<0.05$, Figure 6$)$. In low molecular weight heparin nebulization group, activity of SOD and GSHPx was significantly higher than that in saline nebulization group $(P<0.05$, Figures 4 and 5$)$, and content of MDA

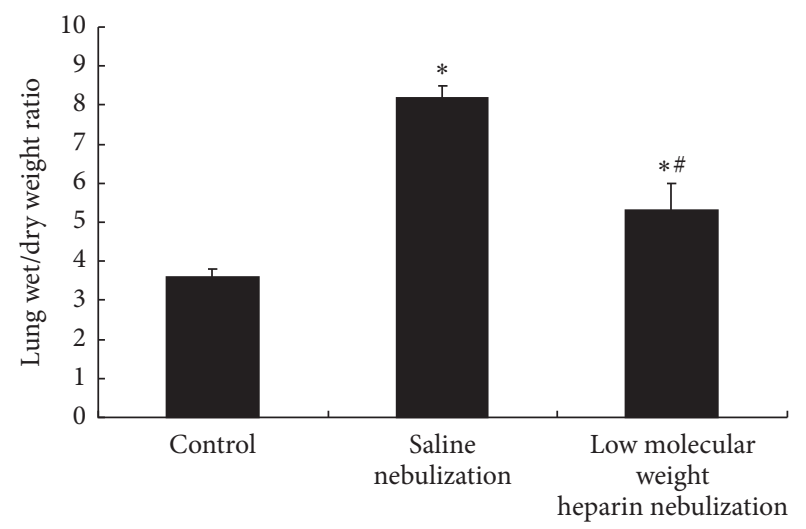

FIGURE 3: Effects of low molecular weight heparin nebulization on ratio of lung wet/dry weight. The middle lobe of the right lung was excised and the wet weight was recorded. The lobe was then placed in an incubator at $80^{\circ} \mathrm{C}$ to obtain the dry weight. The weight ratios were calculated by dividing the wet weight by the dry weight. Data are presented as the mean $\pm \mathrm{SD} .{ }^{*} P<0.05$ versus control group; ${ }^{\#} P<0.05$ versus saline nebulization group.

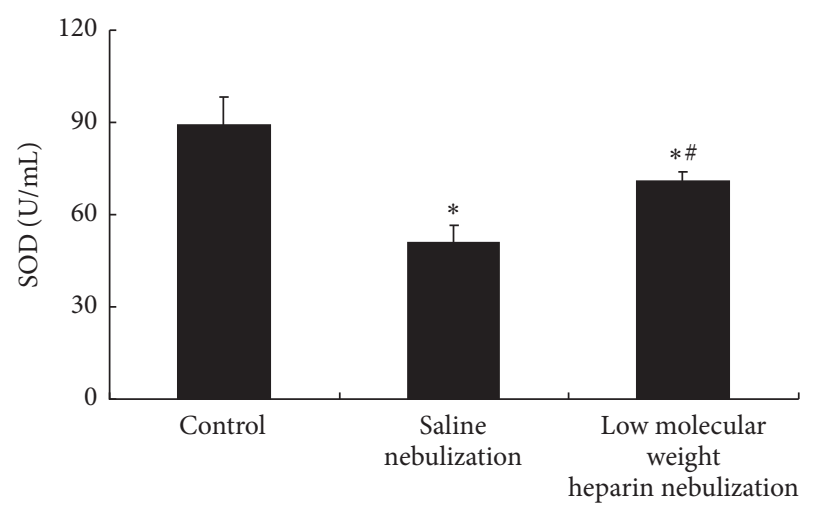

FIGURE 4: Effects of low molecular weight heparin nebulization on SOD activity. The lungs were homogenized in ice-cold phosphate buffered saline and centrifuged. The supernatant was collected to determine the activity of SOD. Data are presented as the mean \pm SD. ${ }^{*} P<0.05$ versus control group; ${ }^{\#} P<0.05$ versus saline nebulization group.

was significantly lower than that in saline nebulization group $(P<0.05$, Figure 6).

3.5. Effects of LMWH Nebulization on Akt Phosphorylation. Because the Akt phosphorylation is associated with oxidative stress response, we subsequently examined the effects of LMWH nebulization on Akt phosphorylation by ALI to explore the potential molecular mechanisms. The Akt phosphorylation on Ser473 was measured by Western blot and the data showed that the endogenous level of phosphoAkt expression (Ser473) in both saline nebulization group and LMWH nebulization group increased significantly $(P<$ 0.05 , Figure 7), while that in LMWH nebulization group was downregulated compared to that in saline nebulization group $(P<0.05$, Figure 7$)$. The ALI related Akt phosphorylation was attenuated by LMWH nebulization. 


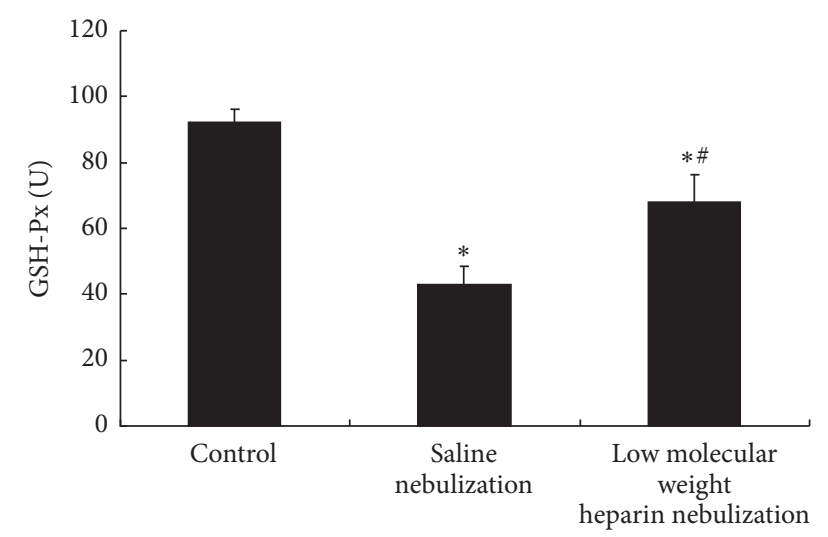

FIGURE 5: Effects of low molecular weight heparin nebulization on GSH-Px activity. The lungs were homogenized in ice-cold phosphate buffered saline and centrifuged. The supernatant was collected to determine the activity of GSH-Px. Data are presented as the mean \pm SD. ${ }^{*} P<0.05$ versus control group; ${ }^{*} P<0.05$ versus saline nebulization group.

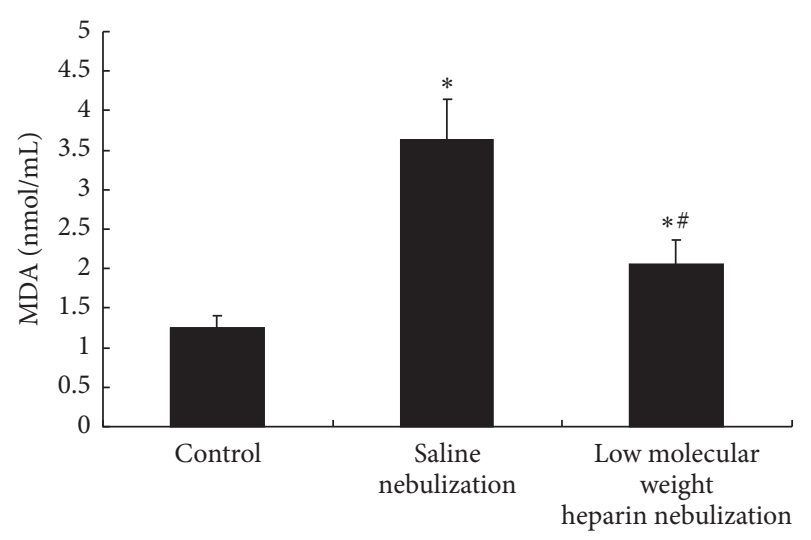

FIGURE 6: Effects of low molecular weight heparin nebulization on MDA content. The lungs were homogenized in ice-cold phosphate buffered saline and centrifuged. The supernatant was collected to determine the MDA content. Data are presented as the mean \pm SD. ${ }^{*} P<0.05$ versus control group; ${ }^{\#} P<0.05$ versus saline nebulization group.

\section{Discussion}

As clotting disorder is one of the features of ALI, anticoagulants such as heparin may possibly have a certain therapeutic effect [9]. Our data showed that $\mathrm{PaO}_{2}$ was increased and lung wet/dry weight ratio as well as total protein content in BALF was decreased after the ALI rabbits were treated by LMWH nebulization, indicating that LMWH nebulization may improve pulmonary gas exchange, increase oxygenation index and decrease alveolar exudation, and therefore attenuate acute lung injury to some extent. Consistently, Li et al. found that lung wet/dry weight ratio in lung injury model of mice induced by high-tidal-volume ventilation was decreased by low-dose unfractionated heparin and enoxaparin, but they did not examine the effects of LMWH and the drugs were given subcutaneously [10]. We currently used LMWH by

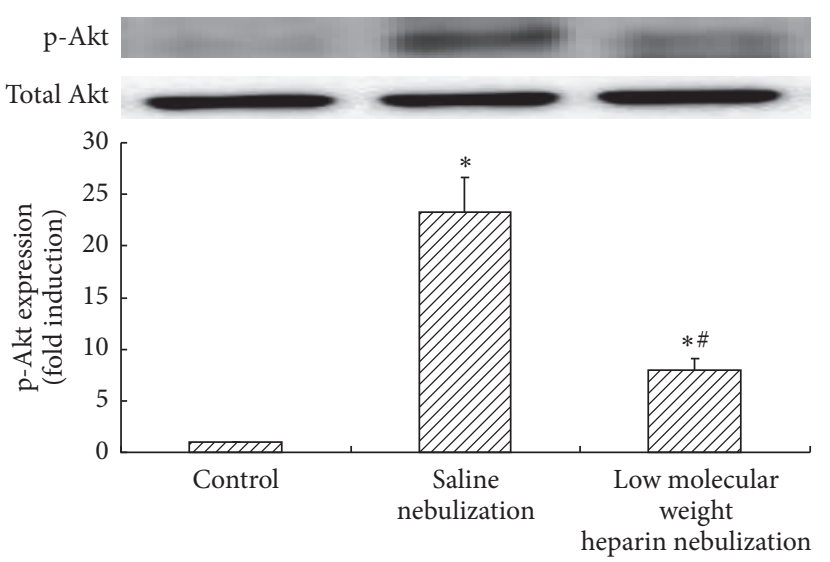

FIGURE 7: Effects of low molecular weight heparin nebulization on Akt phosphorylation. The lungs were homogenized in lysis buffer and centrifuged. The cell lysates were collected and the expression of Akt phosphorylation (p-Akt) and Akt total protein (Total Akt) was determined by Western blot. Level of p-Akt was quantitated by densitometry and plotted as fold induction. Data are presented as the mean \pm SD. ${ }^{*} P<0.05$ versus control group; ${ }^{\#} P<0.05$ versus saline nebulization group.

the dosing method of nebulization and also confirmed the therapeutic effects on ALI.

Furthermore, we tried to explore the underlying mechanism of the therapeutic effects of LMWH nebulization on ALI. It is reported that lung oxidative stress injury was aggravated in ALI [11-13]. Our results showed that the activity of SOD and GSH-Px was significantly decreased while the content of MDA was significantly increased in ALI, indicating that the traumatic ALI can lead to lung oxidative stress reaction. After the application of LMWH nebulization therapy, the SOD and GSH-Px activity was rebounded and the increase of MDA content was significantly inhibited, indicating that LMWH nebulization can play a role of antioxidant, reduce oxidative stress reaction, and attenuate the ALI. Rajeswari and Varalakshmi also found that LMWH has antioxidant potential in countering the oxidative challenge in the experimental oxidative renal injury model of rats [14].

Since oxidative stress can lead to the Akt phosphorylation $[15,16]$, it is important to explore whether the enhancement of oxidative stress caused by ALI in the current study is associated with the Akt phosphorylation. Lee et al. found that ALI caused by LPS can be alleviated through the inhibition of Akt phosphorylation in mice, indicating that the Akt phosphorylation has certain correlation with the severity of ALI [17]. Our results showed that phosphorylated Akt protein expression was increased in ALI, suggesting the Akt phosphorylation might be involved in the development of ALI. This was consistent with the changes of SOD, GSH-Px activity, and the MDA content detected in this study, indicating that the Akt phosphorylation is possibly involved in ALI related oxidative stress reaction.

As heparin was found to have antioxidant effects $[18,19]$ and the Akt phosphorylation was involved in oxidative stress, we therefore tried to detect whether LMWH has influence on 
the Akt phosphorylation. Li et al. found that LMWH administrated subcutaneously can reduce hyperoxia-augmented ventilator-induced ALI in mice through the inhibition of Akt phosphorylation [20]. Our current study found that Akt phosphorylation was restrained in ALI rabbits after LMWH nebulization therapy. At the same time, the changes of oxidative stress indicators were eased, indicating that $\mathrm{LMWH}$ nebulization might inhibit the Akt phosphorylation to reduce the levels of oxidative stress, so as to mitigate the effects of ALI and play a therapeutic role.

In conclusion, the present study found that LMWH nebulization treatment can relieve the traumatic ALI in rabbits, improve lung tissue activity of SOD and GSH-Px, reduce MDA content, and inhibit oxidative stress possibly by suppressing the Akt phosphorylation. This study discussed the effects of LMWH nebulization in the treatment of ALI with new delivery methods from the point of view of inhibiting oxidative stress. Also this study illustrated the related mechanism and offered experimental basis for the clinical treatment of ALI.

\section{Conflicts of Interest}

The authors declare that they have no conflicts of interest.

\section{Authors' Contributions}

Nianlin Xie and Menglei Huan contributed equally to this work.

\section{References}

[1] M. D. Zilberberg and S. K. Epstein, "Acute lung injury in the medical ICU: comorbid conditions, age, etiology, and hospital outcome," American Journal of Respiratory and Critical Care Medicine, vol. 157, no. 4, part 1, pp. 1159-1164, 1998.

[2] L. B. Ware and M. A. Matthay, "The acute respiratory distress syndrome," The New England Journal of Medicine, vol. 342, no. 18, pp. 1334-1349, 2000.

[3] P. Dahlem, A. P. Bos, J. J. Haitsma, M. J. Schultz, J. C. M. Meijers, and B. Lachmann, "Alveolar fibrinolytic capacity suppressed by injurious mechanical ventilation," Intensive Care Medicine, vol. 31, no. 5, pp. 724-732, 2005.

[4] P. Dahlem, A. P. Bos, J. J. Haitsma et al., "Mechanical ventilation affects alveolar fibrinolysis in LPS-induced lung injury," European Respiratory Journal, vol. 28, no. 5, pp. 992-998, 2006.

[5] E. Al-Ansari, H.-K. Du, L. Yu et al., "Low-molecular-weight heparin inhibits hypoxic pulmonary hypertension and vascular remodeling in guinea pigs," Chest, vol. 132, no. 6, pp. 1898-1905, 2007.

[6] J. Han, R. Ding, D. Zhao, Z. Zhang, and X. Ma, "Unfractionated heparin attenuates lung vascular leak in a mouse model of sepsis: role of RhoA/Rho kinase pathway," Thrombosis Research, vol. 132, no. 1, pp. e42-e47, 2013.

[7] E. Mu, R. Ding, X. An, X. Li, S. Chen, and X. Ma, "Heparin attenuates lipopolysaccharide-induced acute lung injury by inhibiting nitric oxide synthase and TGF- $\beta /$ Smad signaling pathway," Thrombosis Research, vol. 129, no. 4, pp. 479-485, 2012.

[8] M. Wang, J. He, B. Mei, X. Ma, and Z. Huo, "Therapeutic effects and anti-inflammatory mechanisms of heparin on acute lung injury in rabbits," Academic Emergency Medicine, vol. 15, no. 7, pp. 656-663, 2008.

[9] C. Feistritzer and C. J. Wiedermann, "Effects of anticoagulant strategies on activation of inflammation and coagulation," Expert Opinion on Biological Therapy, vol. 7, no. 6, pp. 855-870, 2007.

[10] L. F. Li, C. C. Huang, H. C. Lin, Y. H. Tsai, D. A. Quinn, and S. K. Liao, "nfractionated heparin and enoxaparin reduce highstretch ventilation augmented lung injury: a prospective, controlled animal experiment," Critical Care, vol. 13, no. 4 , article R108, 2009.

[11] D. Gokcinar, V. Ergin, A. Cumaoglu, and A. Menevse, "Effects of ketamine, propofol, and ketofol on proinflammatory cytokines and markers of oxidative stress in a rat model of endotoxemiainduced acute lung injury," Acta Biochimica Polonica, vol. 60, no. 3, pp. 451-456, 2013.

[12] C. F. Ronchi, A. L. A. Ferreira, F. J. Campos et al., "Interactive effects of mechanical ventilation, inhaled nitric oxide and oxidative stress in acute lung injury," Respiratory Physiology and Neurobiology, vol. 190, no. 1, pp. 118-123, 2014.

[13] C. Wang, Q. Huang, C. Wang et al., "Hydroxysafflor yellow A suppress oleic acid-induced acute lung injury via protein kinase A," Toxicology and Applied Pharmacology, vol. 272, no. 3, pp. 895-904, 2013.

[14] A. Rajeswari and P. Varalakshmi, "Low molecular weight heparin protection against oxalate-induced oxidative renal insult," Clinica Chimica Acta, vol. 370, no. 1-2, pp. 108-114, 2006.

[15] N. A. Umoh, R. K. Walker, M. Al-Rubaiee, M. A. Jeffress, and G. E. Haddad, "Acute alcohol modulates cardiac function as PI3K/Akt regulates oxidative stress," Alcoholism-Clinical and Experimental Research, vol. 38, no. 7, pp. 1847-1864, 2014.

[16] C. H. Park, D. H. Kim, M. H. Park et al., "Chinese prescription Kangen-karyu and Salviae Miltiorrhizae Radix improve agerelated oxidative stress and inflammatory response through the PI3K/Akt or MAPK pathways," American Journal of Chinese Medicine, vol. 42, no. 4, pp. 987-1005, 2014.

[17] J.-P. Lee, Y.-C. Li, H.-Y. Chen et al., "Protective effects of luteolin against lipopolysaccharide-induced acute lung injury involves inhibition of MEK/ERK and PI3K/Akt pathways in neutrophils," Acta Pharmacologica Sinica, vol. 31, no. 7, pp. 831838, 2010.

[18] L. Dzieciuchowicz, P. Checinski, and H. Krauss, "Heparin reduces oxidative stress in the postoperative period," Medical Science Monitor, vol. 8, no. 9, pp. CR657-CR660, 2002.

[19] P. Finotti, A. Pagetta, and T. Ashton, "The oxidative mechanism of heparin interferes with radical production by glucose and reduces the degree of glycooxidative modifications on human serum albumin," European Journal of Biochemistry, vol. 268, no. 8, pp. 2193-2200, 2001.

[20] L.-F. Li, C.-T. Yang, C.-C. Huang, Y.-Y. Liu, K.-C. Kao, and H.-C. Lin, "Low-molecular-weight heparin reduces hyperoxiaaugmented ventilator-induced lung injury via serine/threonine kinase-protein kinase B," Respiratory Research, vol. 12, article 90, 2011. 


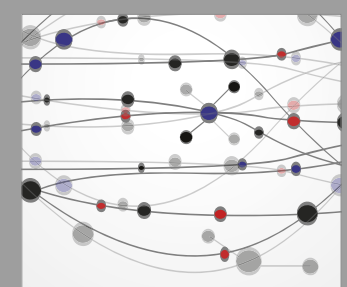

The Scientific World Journal
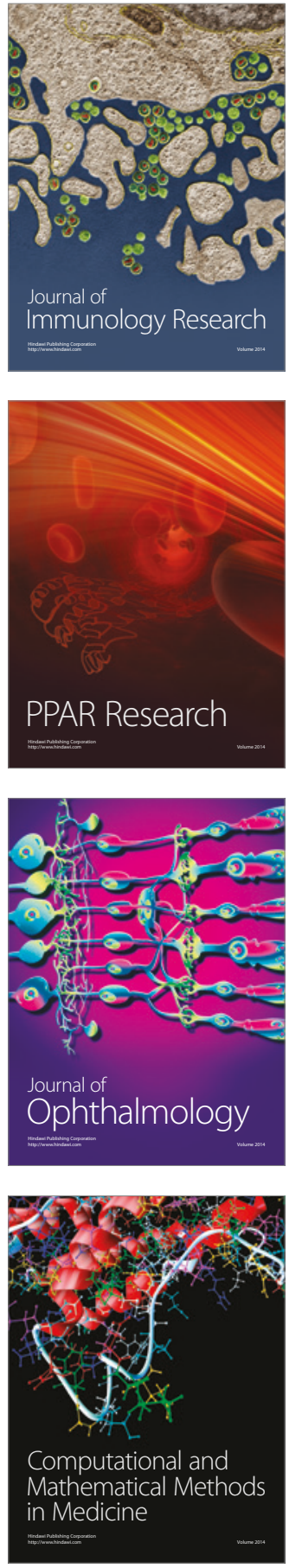

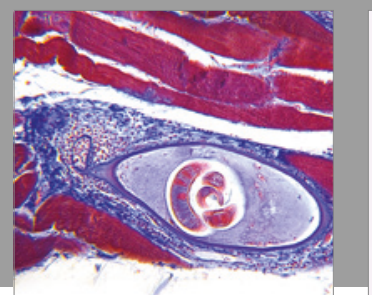

Gastroenterology Research and Practice
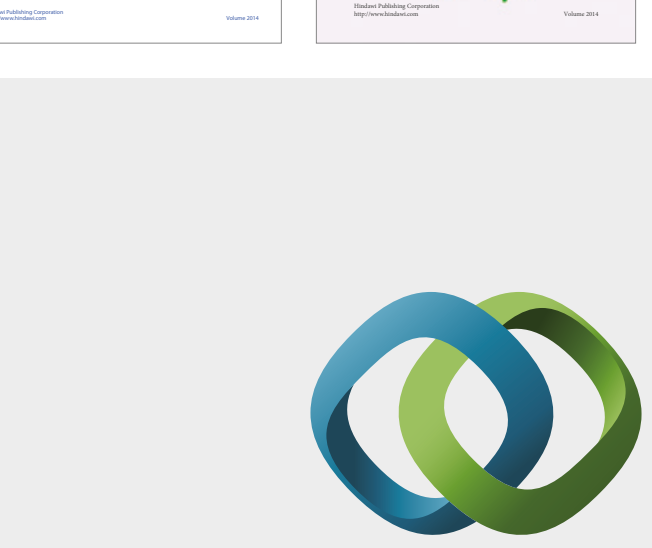

\section{Hindawi}

Submit your manuscripts at

https://www.hindawi.com
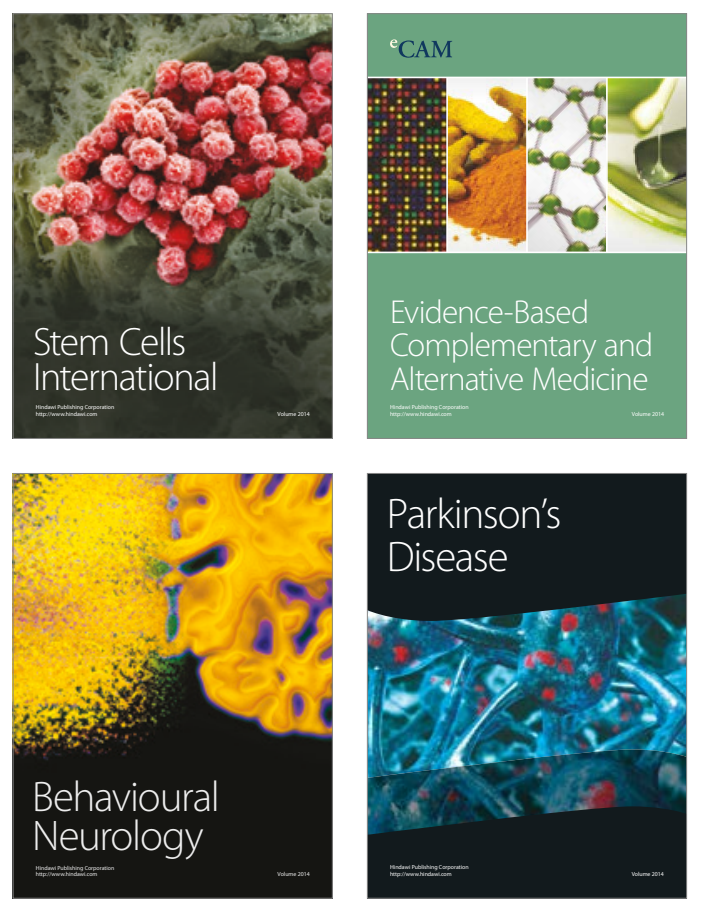
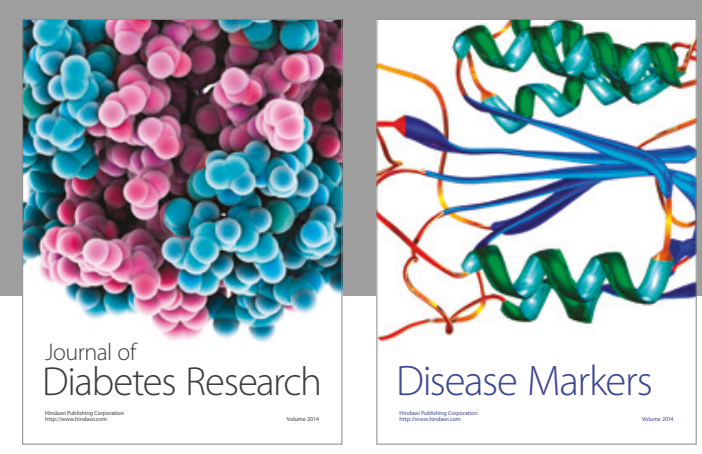

Disease Markers
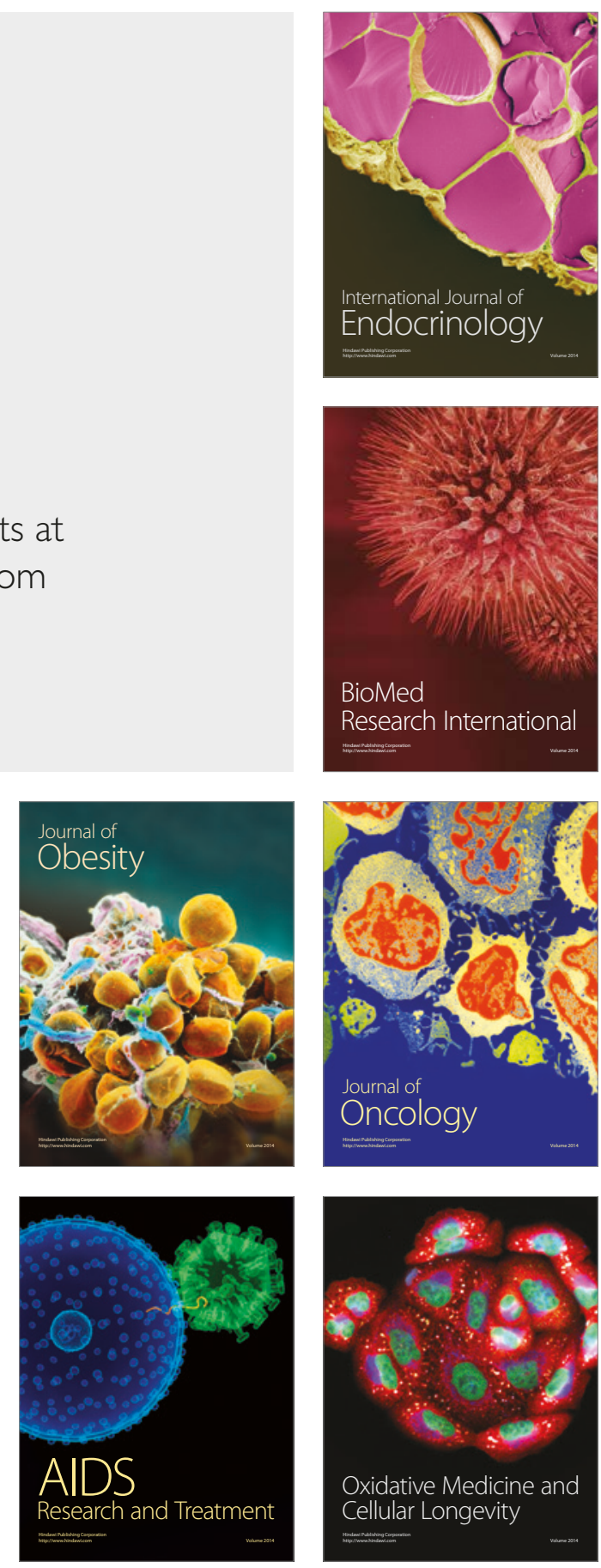